\title{
GRISLEE: Gasmain Repair \& Inspection System for Live Entry Environments
}

\author{
Hagen Schempf \\ Edward Mutschler \\ Vitaly Goltsberg \\ hagen+@cmu.edu \\ edm@rec.ri.cmu.edu \\ vitaly@rec.ri.cmu.edu \\ William Crowley \\ crowley@ri.cmu.edu \\ Carnegie Mellon University \\ Robotics Institute \\ 5000 Forbes Ave. \\ Pittsburgh, PA 15213
}

\section{ABSTRACT}

US gas companies spend over $\$ 300$ million annually detecting and repairing gas leaks in urban and suburban settings. The current approach is one of above ground leak detection and pinpointing, followed by excavation, repair and restoration. The major cost incurred is typically that of digging and restoring the excavation site. The Gas Research Institute (GRI) and the National Aeronautics and Space Administration (NASA) are funding a program at Carnegie Mellon University's (CMU) Robotics Institute (RI) and Maurer Engineering (MEI) to reduce the cost of repairing gas distribution mains using advanced remote and robotics technologies to provide up to 50\% cost savings over conventional repair methods. Under this program, CMU has developed GRISLEE (Gasline Robotic Inspection System for Live Entry Environments), a coiled-tubing deployed, remotely controllable, modular leak-detection, inspection, surface-preparation and repair robot system for the real-time in-situ spot-repair of live 4inch diameter distribution gas-mains. The system is capable of repairing 2 or more leaks per day from a single excavation over a 2,000 foot length under live conditions (i.e. without downtime of the gas main and thus no customer service interruption). The prototype system has undergone laboratory testing and proven the feasibility of its modular inspection, sensing, preparation, and repair technologies and systems. Field-trials with multiple utilities will be carried out during late 2002 .

\section{INTRODUCTION}

The natural gas pipeline industry consists of transmission and distribution companies. These pipeline systems can be simple or complicated. However, all gas pipeline companies are held to the same safety standards. Figure 1 represents one of the many possible configurations of natural gas transmission and distribution systems typically found in the US and worldwide. Note that connectionlines denoted as pipelines are not to scale and can signify thousands of miles (cross-country pipelines) and highly deviated and interconnected networks (urban areas).

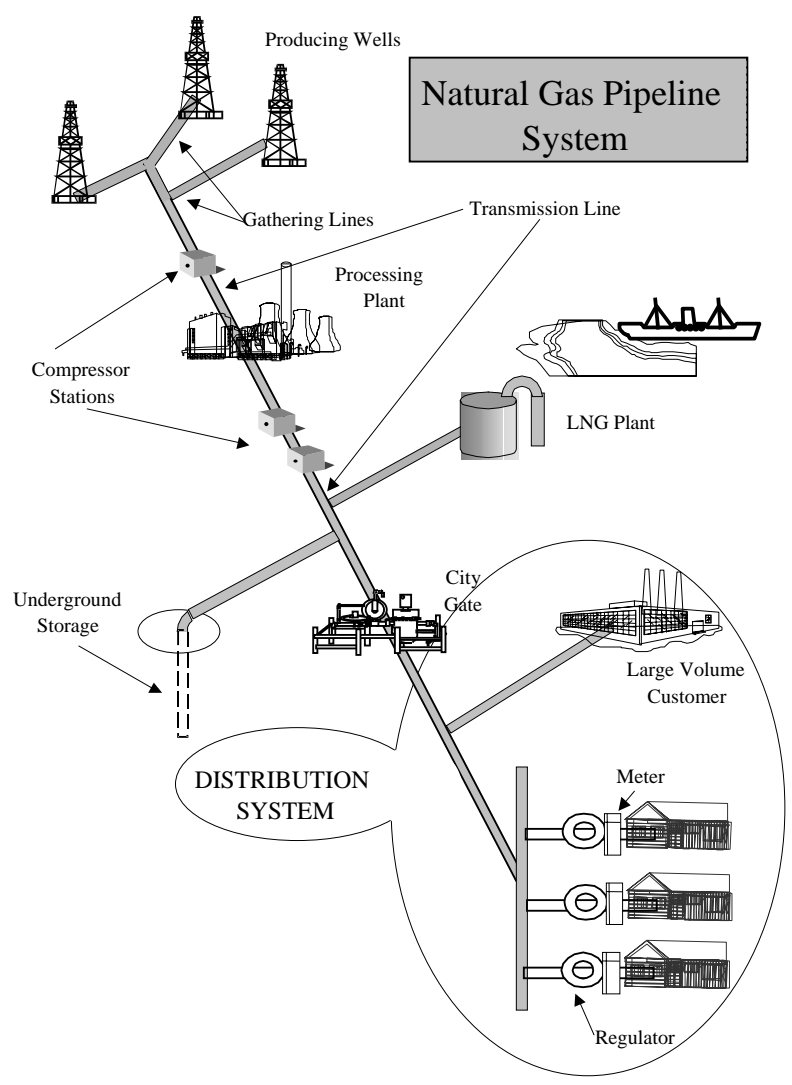

Figure 1 : Overview of gas-production to consumer lifeline

In order to understand natural gas flow, one can envision that the natural gas:

- Flows from the producing wells into gathering line(s).

- Through gathering lines and compressors or compressor stations.

- After the compressor(s), through transmission lines.

- To a processing plant; where the heavy ends, such as propane, butane, ethane or natural gasoline, which are initially components of the gas stream, are removed.

- Through the transmission line and additional compressors.

- From the compressors to underground storage or a Liquefied Natural Gas (LNG) plant (where natural gas 
is liquefied by reducing its temperature to minus 260 degree Fahrenheit), or directly to a city gate station or master meter system.

A distribution system that consists of mains and services operating at different pressures, which are controlled by regulators, is schematically depicted in Figure 2.

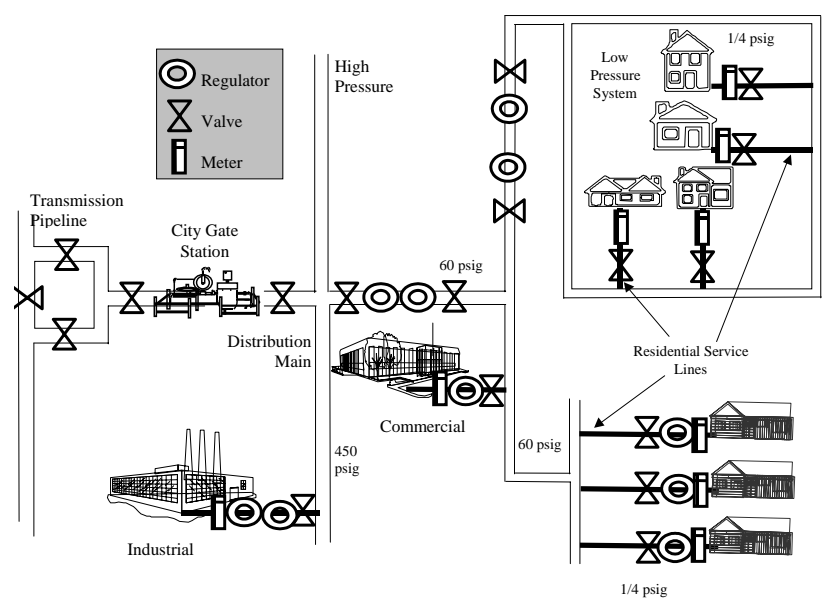

Figure 2 : Distribution system schematic

Ordinarily, industrial customers receive gas service through high-pressure distribution mains, while small commercial and residential gas systems can be either lowor high-pressure distribution systems.

Just like many other commodities supplied to end-users (water, electricity, etc.), natural gas represents a vital component of the human comfort/survival of daily life, whether this be for heating, cooking, power-generation or other uses. Natural gas is delivered to the ultimate enduser through a network of pipelines, both (inter)continental (termed transmission-lines) and local (termed distribution-lines). These distribution lines have been installed in cities over decades and have followed the trends of the latest technologies available on the market at the time - ranging from lead-jointed hollow wooden lines, through cast-iron (low-pressure) to steel (medium to higher pressure) and lately plastic lines.

Typically, the greatest hazard and usually the largest contributor to potentially dangerous situations, are thirdparty damage, caused by inadvertent main-strikes from excavation equipment, resulting in explosive or combustible situations. However, these incidents are rare, and despite being costly, do not necessarily represent the only operational need for safeguarding the gasmain distribution infrastructure in urban settings.

\section{PROBLEM STATEMENT}

Leak and repair crews in all utilities are busy throughout the year responding to gas-detection call-ins from customers, detecting and locating the leak and classifying it into several urgency-classes. Currently, between 800,000 to $1,000,000$ leak-repairs are carried out per year in the US, at a (national-average) cost of between $\$ 750$ to $\$ 1,250$ each, including leak-detection and -pinpointing, excavation, repair and road-restoration (costs are highly variable). The process usually involves the use of sensitive gas-detection equipment to locate, pinpoint the leak [2], mark, and excavate the site around the pipe, verify the leak, apply an external patch (clamp or weld-on), verify the seal, and then restore the excavation/street/sidewalk. Most of these steps (excluding locating and pinpointing) are pictorially rendered in Figure 3.

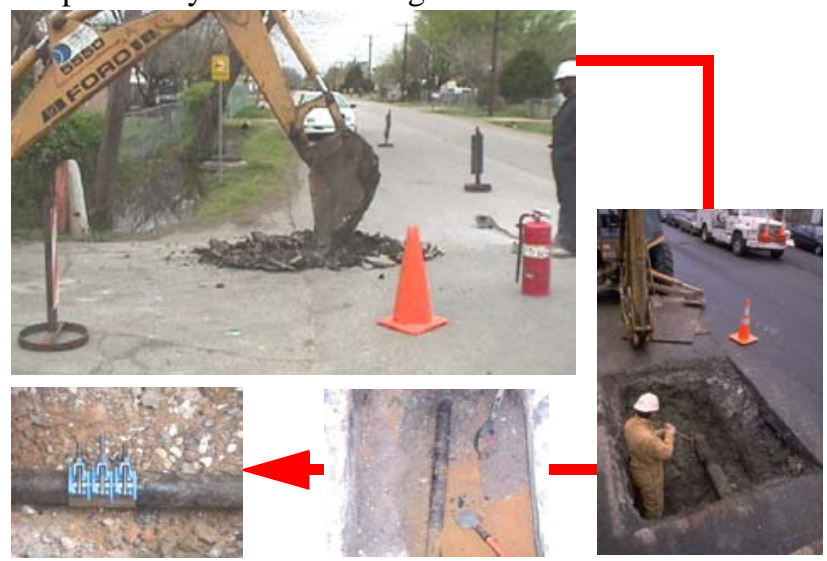

Figure 3 : Conventional leak-repair method

The main issue with manual leak-repair centers on the costs of excavation and the repair-system. If a gas main section is to be relined or replaced, there would be the need to shut off gas-flow to customers on the local grid. In highly urban areas, especially in larger cities with high population densities, the number of inconvenienced customers during the outage, the logistics involved in planning and notifying residents, etc. can be a burdensome and involved activity for utilities.

The utilities were interested in the usage of robotics to provide a lower-cost alternative to pipeline repair and thus extend their usable life or Mean-Time-Between-Repairs (MTBR), providing an alternative to relining and complete replacement (typically with plastic pipe). Utilities thus sought to develop a remote repair system, capable of entering a live gas main without requiring gasflow interruption and capable of inspecting and locating leaks and also repairing them from a single excavation with a substantial reach $(>1,000$ feet). Their expectation was that such a system could drastically reduce repaircosts and -frequency, and potentially provide for a 
preventative maintenance-tool as part of the inspection for gas-distribution utilities. The main question was what technology was available and what needed to be developed so as to achieve an optimal combination of system performance and cost-effectiveness.

\section{TECHNOLOGY MATURITY}

There are many examples of prior-art robotic systems for use in underground piping (transmission-pipeline pigs excluded). Most of them however are focussed on water- and sewer-lines, and meant for inspection, repair and rehabilitation (Pearpoint [7], Beaver, KATE [9], ARIES [10], etc.). As such, they are mostly tethered [11], utilize cameras and specialized tooling [14], etc. (see Figure 4).
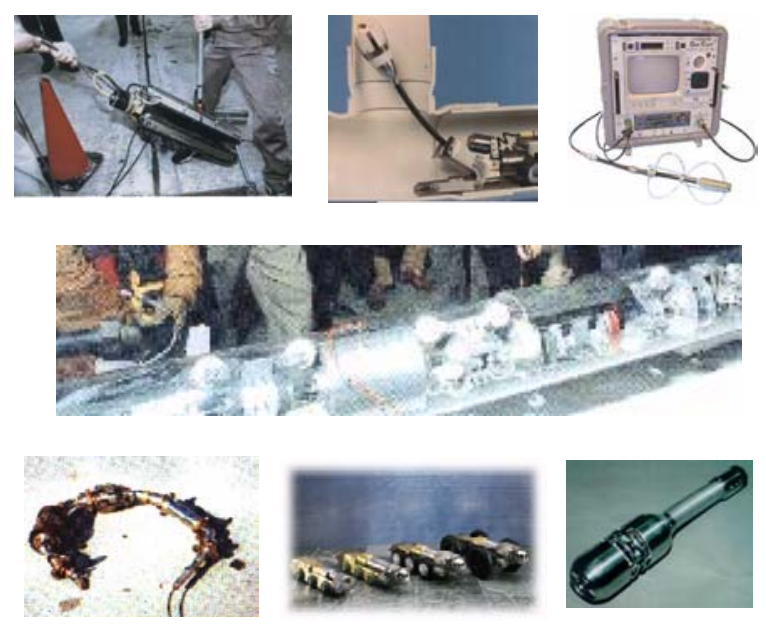

Figure $4:$ Prior art robots in use in the sewer industry
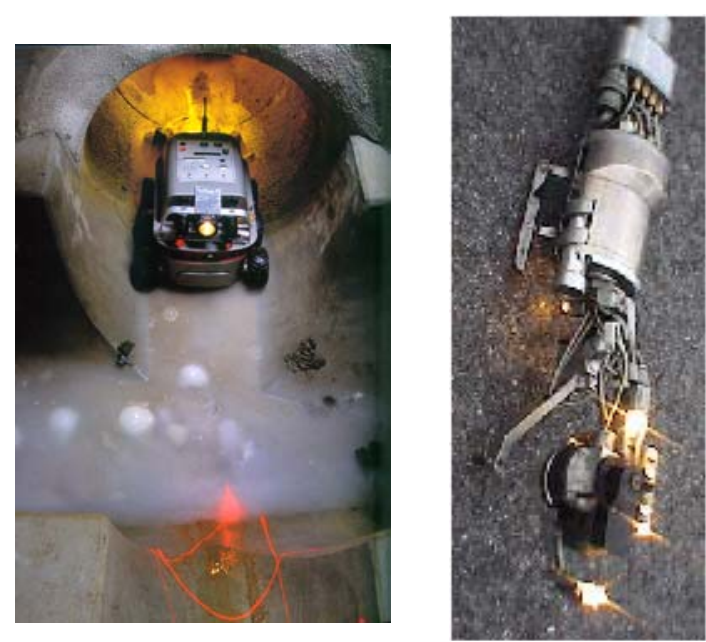

Figure 5 : Tethered gasline (right) and untethered autonomous (Kurt I) robots developed to date by industry and researchers
Two of the more notable exceptions are the autonomous Kurt I system from GMD (Germany; [6]) used for sewer monitoring (not commercial nor hardened), and the (albeit tethered) cast-iron pipe jointsealing robot (CISBOT; [12]), which is deployed through a bolt-on fitting and injects anaerobic sealant into the leaking jute-stuffed joint (These systems are shown in Figure 5).

There are several types of distribution gasmain repair systems commercially available today. The lowpressure repairs (i.e. on cast-iron) are primarily carried out externally, by applying a screw-tightened clamp with an elastomeric seal along a section of pipe and over the leak-spot. High-pressure mains (typically steel) are sealed using a metallic weld-on sleeve, which is also applied externally; both can be applied under live conditions (see Figure 6).

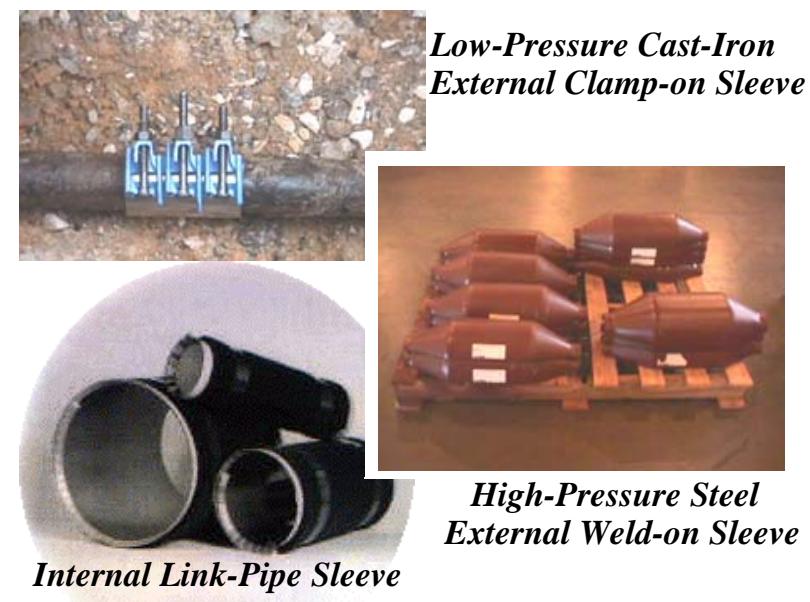

Figure 6 : Existing gasmain repair systems

Internal repairs are either performed with a dead main and (almost exclusively) for joint-repair, using either internally-set epoxy-hardening sleeves [13] or also external (live) fiberglass-epoxy sleeve-clamps (not shown $)^{1}$.

It seemed that none of the existing technology solutions could achieve what utilities were seeking to accomplish in terms of performance and cost-point. It was at that point that the Gas Research Institute (GRI) and NASA contracted Carnegie Mellon University (CMU) and Maurer Engineering, Inc. (MEI) to take on a novel development program.

As part of the program, CMU was tasked to develop a simple, rugged, yet effective repair robot system that could be deployed in a modular fashion and perform the

\footnotetext{
1. Notice that internal joint-sealing with anaerobic sealant jute-injection is not explored here
} 
detection, preparation and area repair/reinforcement of a leaking live gas main. CMU was teamed with Maurer Engineering, Inc. (MEI) to utilize GRI's live-pipe access and coiled tubing (CT) deployment system (structural push-pull steel-tubing 'tether') to deploy their robotic system (to be called GRISLEE); a schematic of the deployment and robot-system are illustrated in Figure 7 below.

The GRISLEE system was envisioned to consist of several independent modules, which were to be deployed utilizing the CT system through a live-access weld-on valve on the gasmain. A topside operator was to monitor a computer/video display that relayed MFL-data and video imagery from the modules through the CT unit (see Figure 7).

\section{REQUIREMENTS \& SPECIFICA- TIONS}

In order to develop the most appropriate system to repair leaks in live gas-mains, it is imperative that the complete system's performance requirements be spelled out as quantitatively as possible. Towards that end, a complete list of requirements was developed and compiled:

- Faster, cheaper and more convenient repair method than is possible with current methods.

- Access and work within live gas-mains from a single excavation and allow maximum travel from a single entry-point in both directions.
- Fit into and pass through 4-inch I.D. steel gas-mains

- Reasonable negotiate bends, debris \& protruding taps

- Operate safely within a natural gas environment

- Identify, mark, acquire, clean and repair the defective area $(s)$

- Install a reliable sealing-patch

- Guaranteed system retrieval under worst-case system failure

- Modularly interchangeable with existing/future

deployment and sensing components

- Easy to operate with minimal and manual operator interactions

Based on the above performance requirements imposed on the design, and an iterative concept development and evaluation process, the robot development team sought to implement the following set of system specifications:

- System to be designed as a multi-module exchangeable work-head system capable of viewing, inspecting, marking, cleaning and repairing pipeleaks.

- Unit to interface to existing coiled-tubing (CT) deployment system from MEI.

- The hard module-diameter not to exceed 3 inches O.D.

- Inert materials (SS) with internal purging and nitrogen pressurization to 100 psig, as well as potting and immersion to be used as safing techniques.

- The repair-head to have a forward-looking live-video camera monitoring system

- The system to have an independent visual flawmarking emplacement (coupled to MFL-head) and detection (on repair head) system.

- The system to be able to fine-position itself using the CT-unit to within +/- 1 inch or better.

- The internal pipe-surface to be cleaned mechanically.

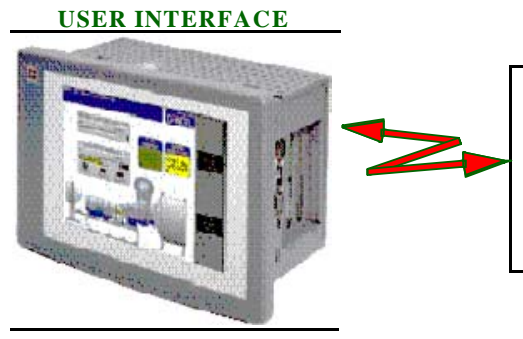

COILED-TUBING UNIT
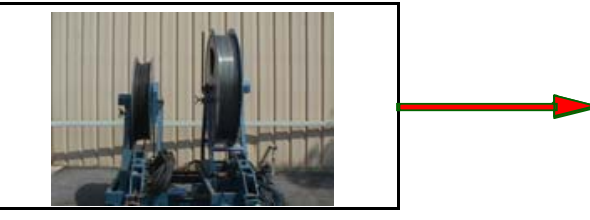

LIVE PIPE-ACCESS SYSTEM

COILED-TUBING PIPING

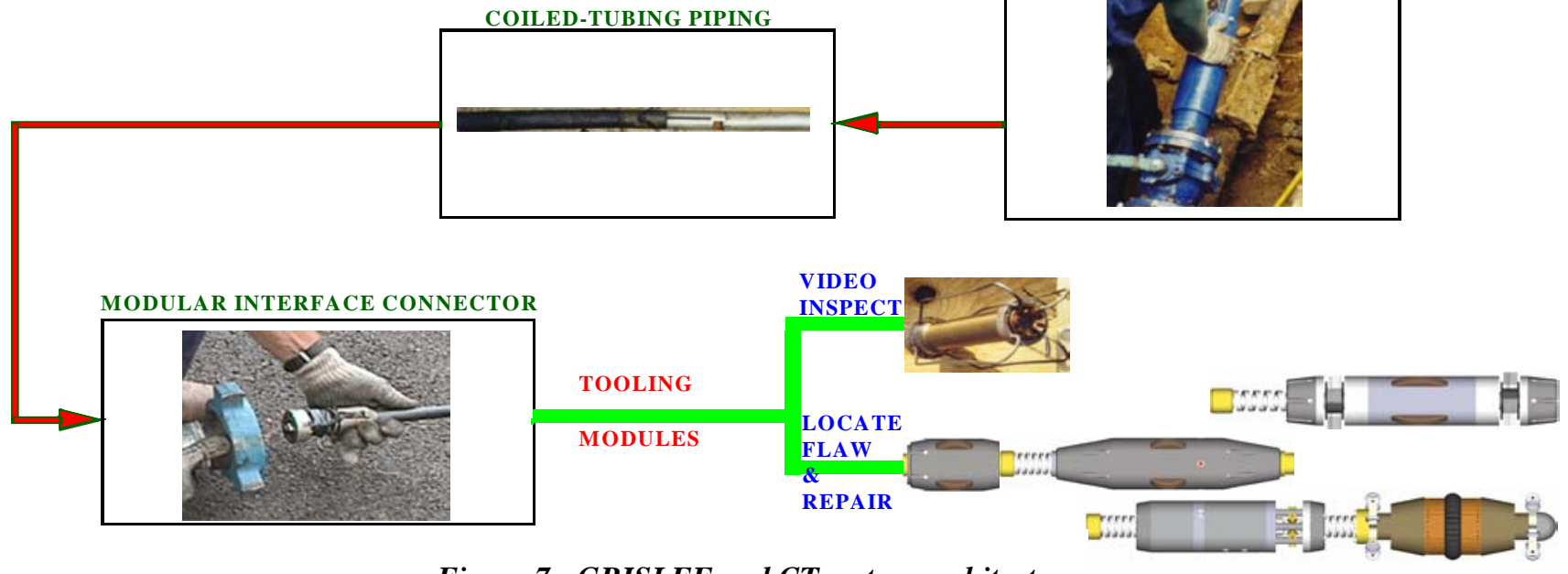

Figure 7 : GRISLEE and CT system architecture 
- The frontal cleaning-head and repair-modules to be interchangeable.

- The operator controls to be integrated with existing CT, MFL and camera controls.

- The system to be deployable in the same manner as the current camera and MFL inspection systems.

In order to resolve 'architectural' issues, a preliminary design-study was undertaken. It revealed that for many technical, operational and logistical reasons (including a time-and-motion study), these modules should be made interchangeable and deployable as individual 'trains', rather than having all modules hooked into a single train inside the pipe.

The utilities also tasked the team to determine whether live main spot-repair from the inside under live conditions with a remote robotic system was cost-effective. CMU and MEI engaged in a preliminary review of a gas-utility's repair-records, and generated relevant data as to the number, frequency and spacing of spot repairs in a suburban distribution network in the Northeastern US. The results are shown in Table 1:

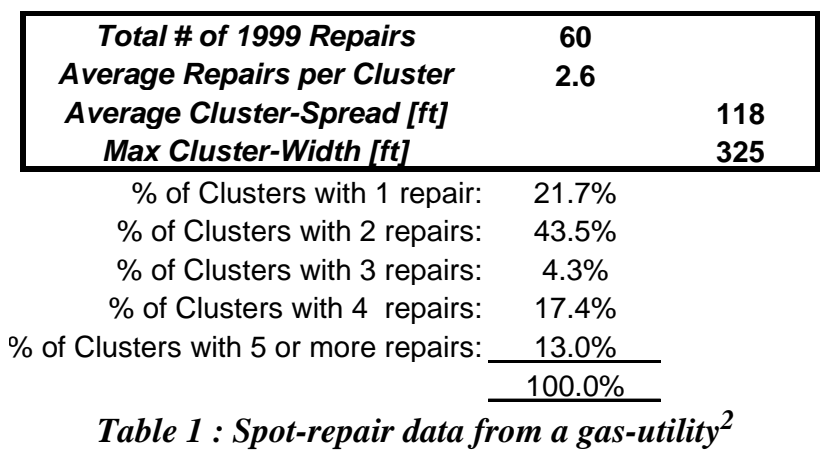

It was found that over a period of one year, repairs carried out in specific areas, tended to result in multiple repairs within certain lengths of pipe. More than $75 \%$ of these repair-jobs patched 2 or more leaks within less than a 325 foot separation between leak-locations. Based on preliminary cost-estimates for live robotic gas main repair, it was determined that if more than 2 repairs could be carried out from a single excavation within a 2,000 foot distance around the excavation, live robotic repair could compete and beat conventional manual methods (based on clamp-on/weld-on sleeves emplaced through open-hole excavation methods). The repair-data and cost-estimates summarized in Figure 8 for both external clamp- and weld-on manual repair sleeves show the payback crossover point. These were the conditions under which the team set out to develop the GRISLEE system.

2. Repair cluster is defined as a section of CTaccessible pipe with a sequence of known leaklocations.

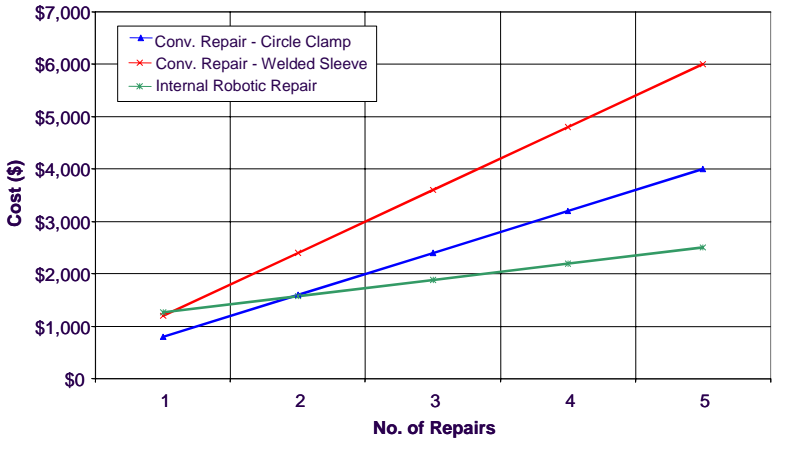

Figure 8 : Payback cross-over for live internal repair

\section{SYSTEM OVERVIEW}

In order to understand the system architecture and then relate to the technical details of each subsystem, it is best to begin the description from the 'topside' location, from where the robotic repair operation was to be undertaken.

The deployment and 'locomotion' unit for the repair system is based solely on a CT-unit ${ }^{3}$. The CT system allows one to access live gas-mains, insert GRISLEE through a welded-on access-fitting, and 'push-pull' GRISLEE through the gas main for distances of up to 1,000 feet in either direction (limited by CT buckling loads and the extent of pipe-bends). GRI and Gaz de France (GDF) had previously funded the development of the CT system (developed through MEI [3]) and the magnetic flux leakage (MFL) flaw-detection sensor-head (developed through TuboScope Vetco Pipeline Services, Inc.).

The team was tasked to develop a set of robotic/remote modules, that would allow a repair crew to: (i) detect wall thinning and/or leaks in the pipe-wall using the existing MFL-sensor, (ii) clean the pipe to prepare the affected pipe-area, and (iii) emplacement of an expandable metallized epoxy-sleeve to reinforce and/or seal and plug the leak under live gas-pressure (inches of water-column to $60 \mathrm{psig}$ ), without affecting the continued gas-flow inside the main line [4]. GRISLEE was designed to be modular and intended to include several modules:

- a camera module to determine obstacles and joint

locations in the gas mains,

- a magnetic flux leakage module to locate and measure remaining steel pipe-wall thickness [3],

- a marker module for marking and locating identified pipe defects,

- a brush module for cleaning a localized area at the defect or joint location, and

- an improved sleeve module to repair leaks in live pressurized gas mains.

3. The $C T$ system is a spooled-up steel-pipe with internal cabling, used extensively in the off-shore oil-industry for sensor-deployment and welltreatment over thousands of feet in depth. 
Depending on the task at hand, the proper arrangement of modules is attached to the flexible whiphose at the end of the CT, and then inserted through the access fitting and pushed to the proper location utilizing the CT system. Once in place, the system is remotely controlled from above ground to perform its task. Upon completion, the module-train is retrieved, and replaced with the proper arrangement of modules to perform the next operation. The proper arrangement of the system architecture is depicted in Figure 7 in an iconic fashion.

Before deploying GRISLEE, the launching apparatus needs to be put in place. This requires (i) the excavation of the pipe-segment in question, (ii) exposing the pipe so as to allow human access to it, (iii) installation of the accessfitting, and (iv) installation of the CT-unit and the launching hardware. GRISLEE is then launched in a predefined sequence, including: (i) attaching the videoinspection train to inspect the pipe for obstructions, (ii) deploying the sensing and marking module-train to utilize the MFL to locate flaws/leaks/joints and then mark them with the marking-module, (iii) insertion of the mechanical brushing system to remove loose scale around the defectarea, (iv) deployment of the spot-repair-sleeve, and finally (v) re-insertion of the camera-module to confirm the proper installation of the sleeve. This process is repeated until all detected and marked flaws are repaired. The same can be done in the opposite direction with a reversed fitting welded to the pipe.

The overall system is best described in terms of its main elements, which are the (A) deployment and access system, and the operator interface, and (B) the robotic inspection/mapping/marking/preparation/repair module trains. The robotic modules are best broken down into their different trains, namely those used for (i) video inspection, (ii) MFL sensing and marking, (iii) surface preparation, and (iv) spot-repair. The electronics, software and user interface are also best described separately.

The access and deployment system developed by MEI under separate GRI and GDF co-funding, consists of a

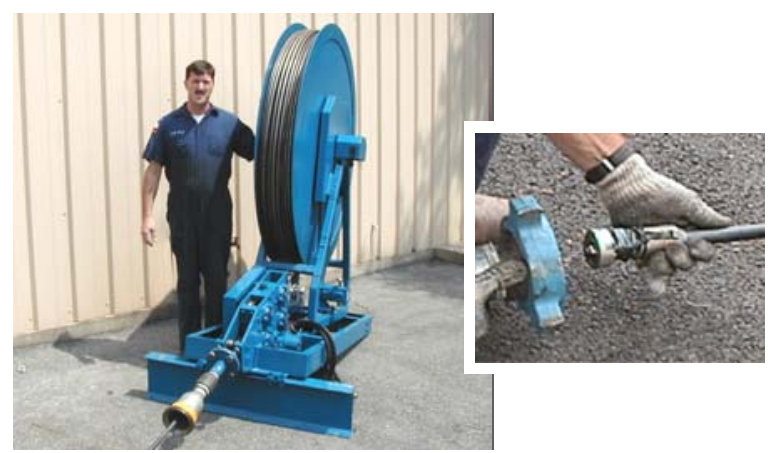

Figure 9 : CT deployment \& connector system diesel-hydraulically powered coiled-tubing (CT) cablespool, wound with mild-steel hollow piping, within which is seated a multi-conductor tether in a pressurized inert nitrogen atmosphere (see Figure 9).

The CT piping is terminated at the spool-end through a multi-conductor slip-ring, that carries data, video and control signals to and from the control panel; at the distal end, a flexible whiphose (reinforced fiber-rod coaxially wound with the multi-conductor tether; see Figure 9) is terminated with a strain-relieved connector.

The access-fitting that is used to allow the CT to be deployed into the live pressurized main, is a two-part welded-on steel-clamp, that has an angled fitting with a valve mounted to it. A hydraulically-powered hole-saw drill is manually deployed to cut an elliptical hole into the top of the pipe to the full diameter of the gasmain, which is then retrieved. After drilling, a launch-chamber is attached to the fitting, which when opened, allows for the attachment of modules and their launch/retrieval without any release of natural gas (Figure 10).
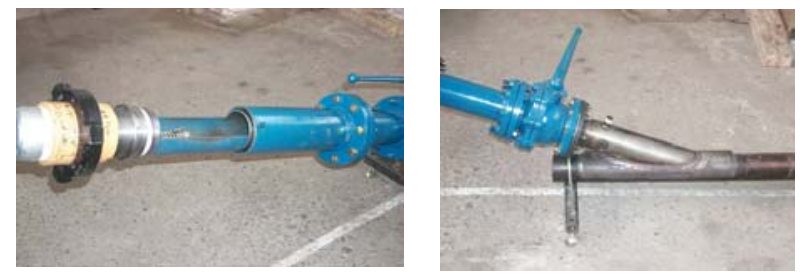

Figure 10 : Live gasmain access and launch fitting

The tooling module-trains (depicted in Figure 11), intended for visual camera-inspection, flaw-detection and -marking, area-preparation and spot-repair, are depicted in Figure 11

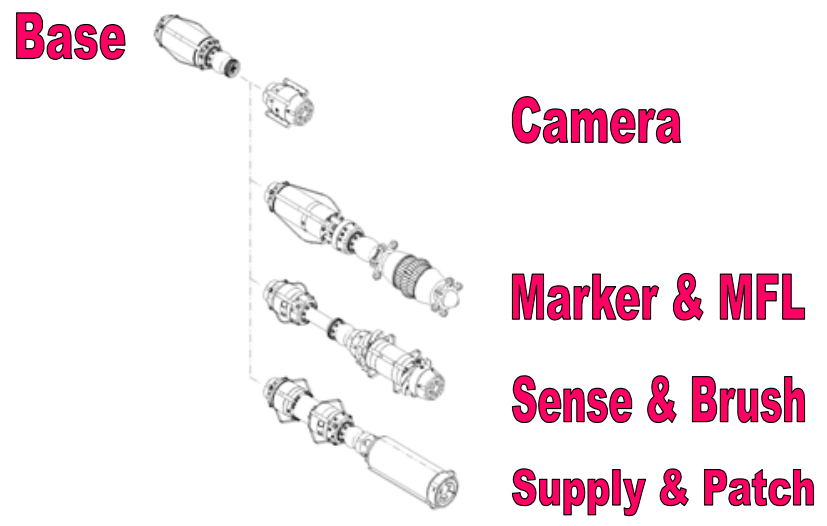

Figure $11: C A D$ view of module-trains

A perspective view of the resulting configurations, as well as a picture of a pre-prototype being test-launched in a 
test-setup, are shown in Figure 12:
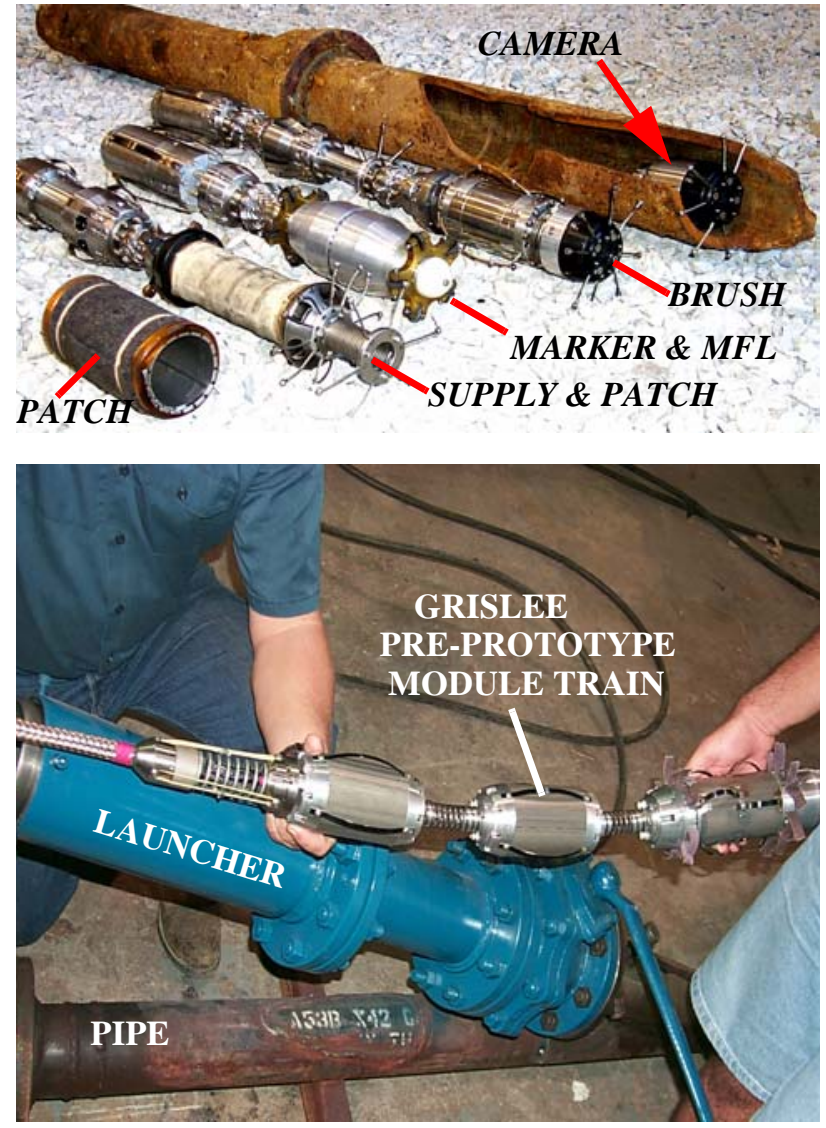

Figure 12 : Train-configurations and pre-prototype testlaunch experiment

The base-module contains the DC power-supply, embedded CPU and serial communication electronics on custom-built circular PCBs that control each module-train, communicate to the topside controller and perform in-situ module-detection and safety-checking. Each modules' hardware safety-line and module-ID line are tied to two separate pins, so that the base can perform a simple faultchecking and module-ID before allowing any powerapplication and controls signal sending.

The video-inspection train is simply a combination of the base-module with a ruggedized camera-module.

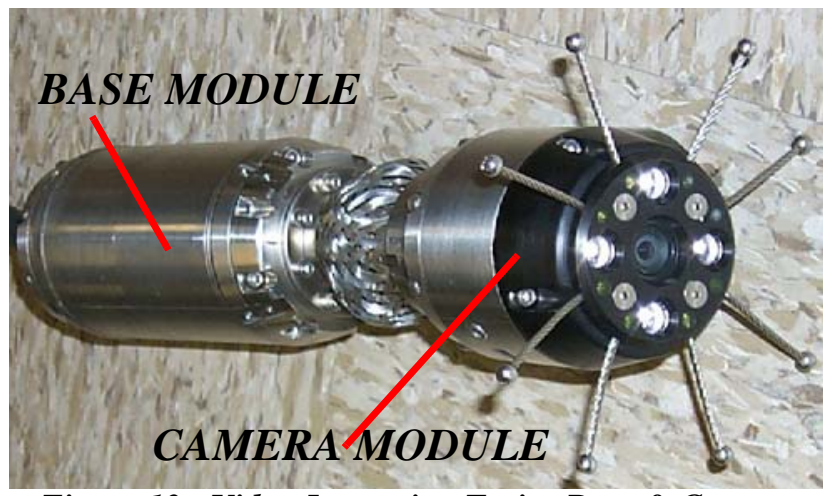

Figure 13 : Video-Inspection Train: Base \& Camera
The camera-module consists of a high-resolution lensed color board-camera with an internally-mounted highintensity white LED light-ring behind protective opticalquality glass-windows. The light-intensity is varied and synched to the video-signal. A view of the combined video-inspection train is shown in Figure 13.

There are several 'non-contact' methods of flaw (cracks, corrosion, etc.) detection available for ferrous pipe materials. The most mature technology is that of Magnetic Flux-Leakage (MFL), where a magnetic field is superimposed by a magnet on the ferrous parent-material, thereby saturating it. The principle behind the MFL sensing technique, is shown in Figure 14. When a magnetic field is applied to a steel-plate (or pipe), magnetic flux-lines go through the conductive metal between the two poles of the magnet (Figure 14-A). These flux lines are 'deformed' if there is a loss of material in the metal surface (saturation effect), causing a localized B-field 'anomaly' (Figure 14-B). This 'anomaly' can be detected by hall-effect sensors (current as a function of the magnetic field applied to the sensor) and mapped as a function of location and signal-strength.
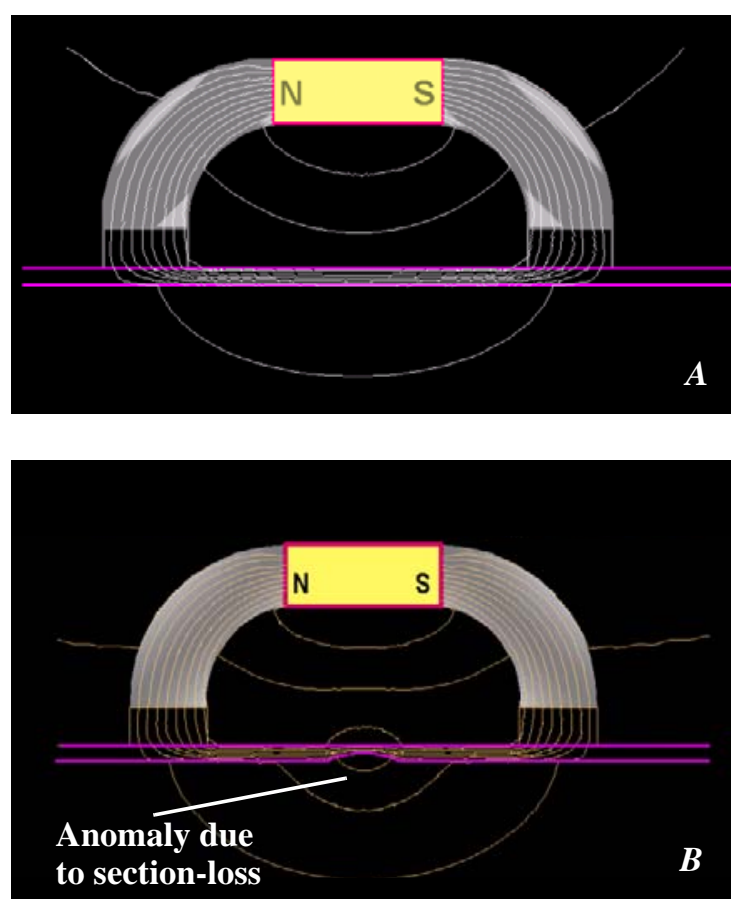

Figure 14 : MFL B-field saturation(-loss) technique

Specifically, a corrosive pipe material-loss will manifest itself as said reduction in the magnetic-flux (or B-field strength). By properly arraying and positioning these halleffect sensors, and polling them, different readings can be obtained, which can be processed by computer to generate a physically meaningful surface-intensity map, which when coupled with false-coloring (C-Scan) can aid in visual identification of cross-sectional material-losses. 
The signal received from hall-effect sensors arrayed in a circular pattern, such as those on the prototype MFL-unit, and the associated visual feedback for the corrosional cross-sectional material loss flaw exhibited by a typical (buried) steel gasmain, are shown in Figure 15:
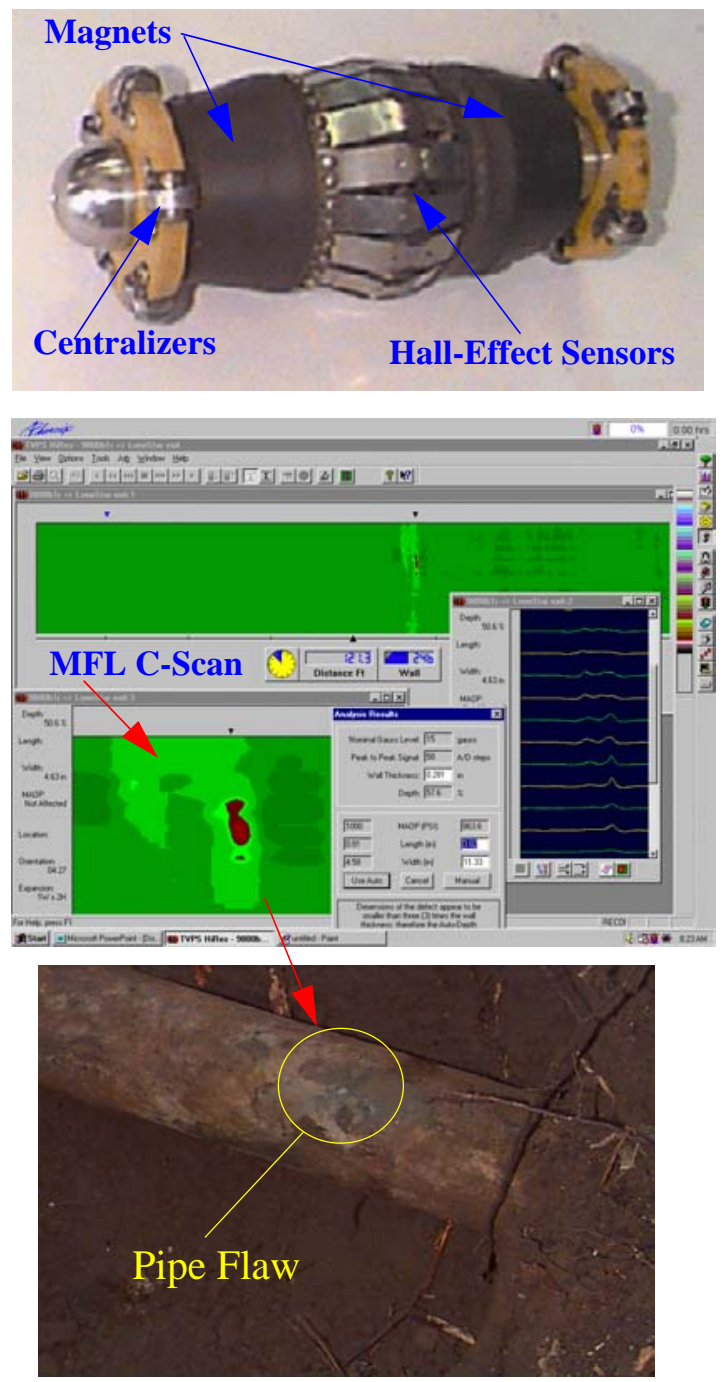

Figure 15 : MLF prototype sensor with graphic feedback on corrosional metal-loss

The marker-module uses internally-pressurized paint through a set of nozzles to mark the inside of the pipe with a visible paint-ring. The goal is to leave a visible mark in the pipe for an optical sensor to detect said mark in later passes. This allows the preparation and repair modules to be accurately positioned without relying solely on odometry-data from the robot or the CT spool. A view of the assembled Marker/MFL train (with a mock-up of the MFL-module) is shown in a cut-up spray-marked pipe in Figure 16.

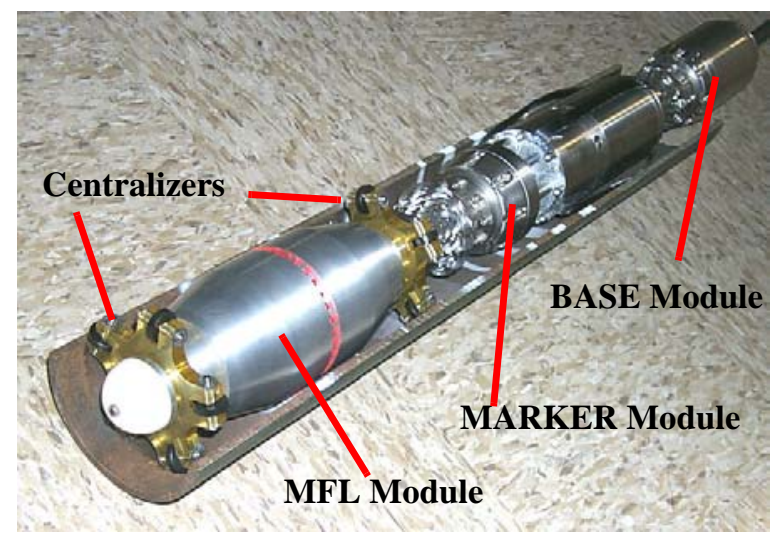

Figure 16: Marker and MFL train in sprayed pipe piece

The method utilized to mark the pipe is based on a timed release of properly-mixed water-based paint through a set of $30^{\circ}$ fan-nozzles. The pneumatic schematic utilized allows also for purging and assuring unclogged lines at all times - see Figure 17:

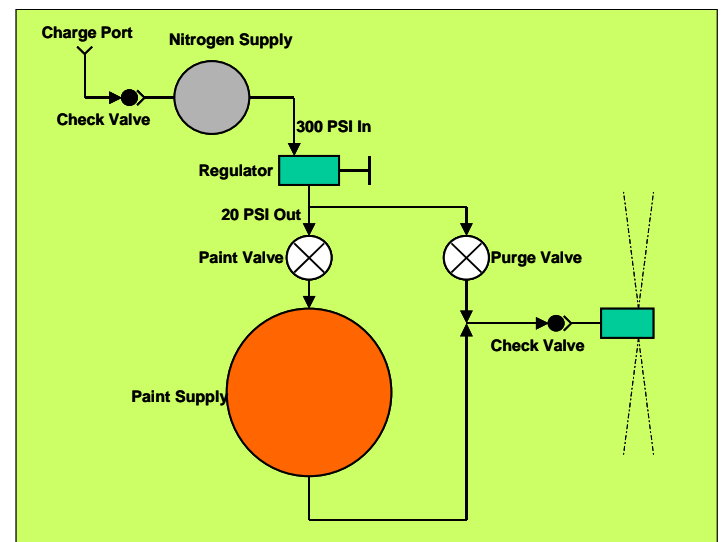

Figure 17 : Pneumatic purging-circuit schematic

The pipe-preparation train consists of the base-, sensorand brush-modules. In order to use them, the operator goes back into the pipe, locates and positions the train over the sprayed-on marker, and then brushes a selectable width of the inside section of the pipe (located so as not to remove the marked paint-strip for later positioning of the repair-module); excessive scale is removed and improves adhesion of the repair-patch. Feedback as to proper position is provided by an integral camera mounted to the front of the brush-module, allowing the operator to do coarse-positioning by extending/retracting the coiledtubing pipe. Fine-positioning is aided by the sensormodule which detects the paint-strip, allowing positioning to within $+/-1 \mathrm{~cm}$. A view of the sensor- and brush- 
module train is shown in Figure 18.

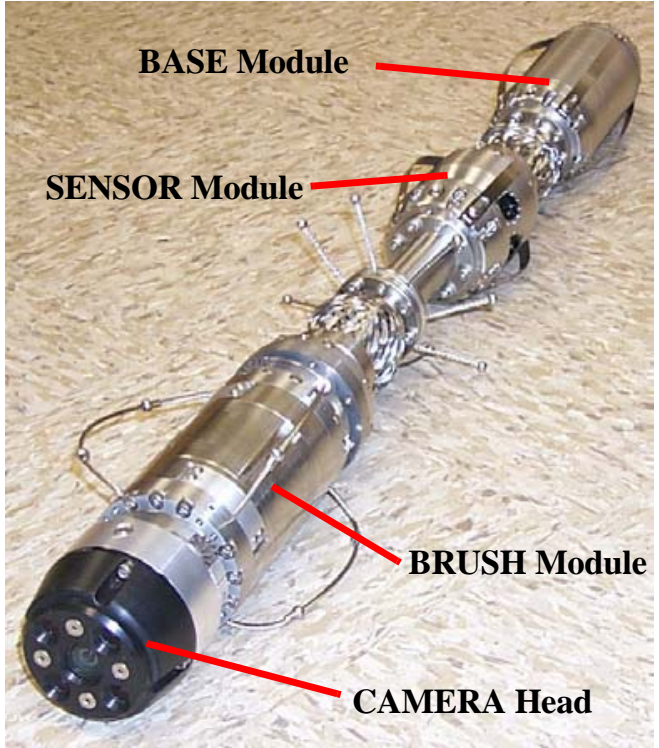

Figure 18 : Sensor- and Brush-module train assembly

The sensor-module contains the electronics and optical sensors necessary to detect the sprayed-on paint ring on the walls of the pipe. The sensors are based on retroreflective infrared detectors giving a simple thresholded binary feedback depending on the intensity of the reflected signal. False-positives are avoided through a customized hardware setup and software algorithm, which runs on the base-module computer.

The brush-module contains the electronics and drivers to activate the motors that power the mechanical surfacetreatment brushes. The driven shaft is designed to allow operators to externally exchange brush-module halves. An internally-mounted camera and light section allows viewing of the prepared area and repeated brushing if needed. The appropriate brushing media depends on the conditions within the pipe. The elements used in impact-, deformation and sanding-operations typical in other surface preparation industries are transferable to this system with minimal effort, and used whenever appropriate. Based on preliminary tests, the impactabrasion method utilized in star-brushes has proven the most effective at removing rust and mill-scale with minimal effort, while exposing raw metal and providing good surface-roughness for the epoxy-based patch to take hold during the setting process.

The repair-train consists of the base-, sensor- and patchmodule. The base-module monitors the sensor-trigger to alert the operator to the proper position of the modules, and controls the valving to inflate and release small amounts of compressed nitrogen into the setting-bladder on the patch-module. The sensor-module is similar to that used on the preparation-train. The patch-module consists of a compressed-nitrogen chamber at $300 \mathrm{psig}$, valved through a regulator to inflate an elastomeric membrane on a setting-pipe. The setting-pipe is hollow, to allow gasflow during the setting-process. An image of the repairtrain is shown in Figure 19.

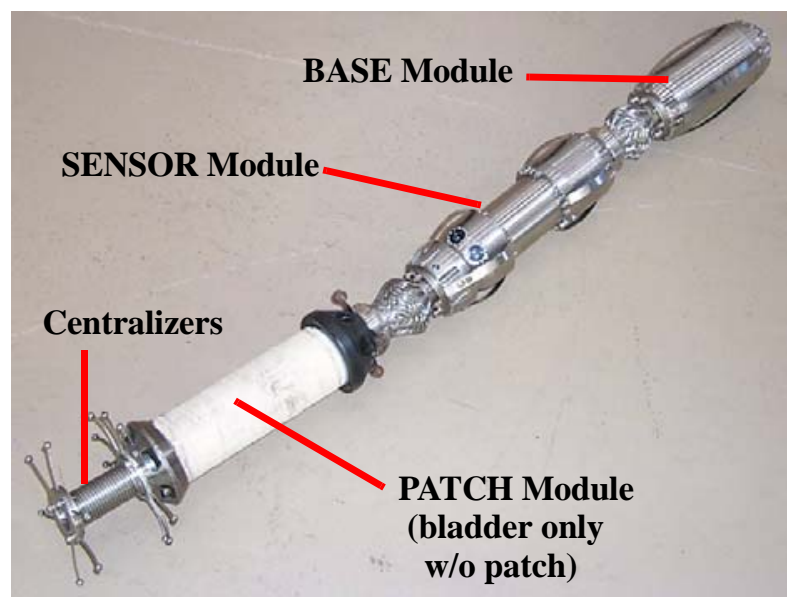

Figure 19 : Repair-train module prototype

The prototype spot-repair patch that was used for the GRISLEE system is a substantially-modified version of the LinkPipe GasSleeve ${ }^{T M}$ spot-repair system developed for repair of out-of-service gas mains. A view of the commercially-available sleeve, prior to modification is shown in Figure 20.

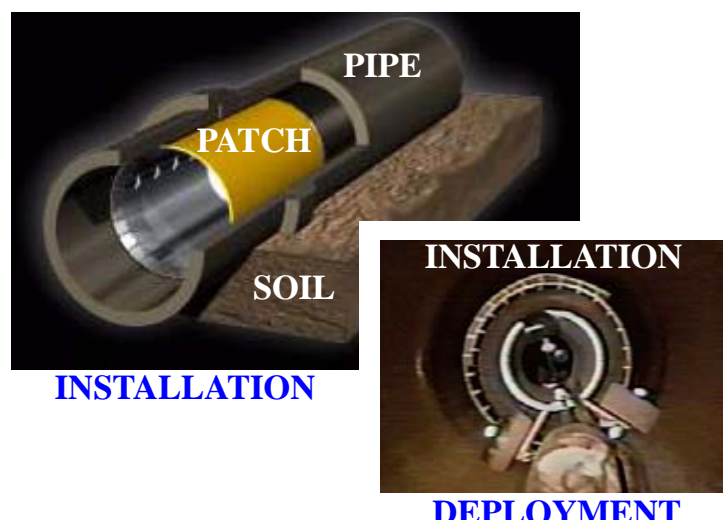

Figure 20 : LinkPipe's Gas-Sleeve ${ }^{\text {TM }}$ spot-repair patch

Modifications to this existing sleeve, included changes to the serration and locking system, as well as the addition of an elastomeric tube to ensure pressure-sealing during the setting-period necessary for the epoxy to set so as to create an impermeable barrier. The prototype system, and the resulting patches in joints and pipes (used clear PVCpipe for better illustration only), are shown in Figure 21. 

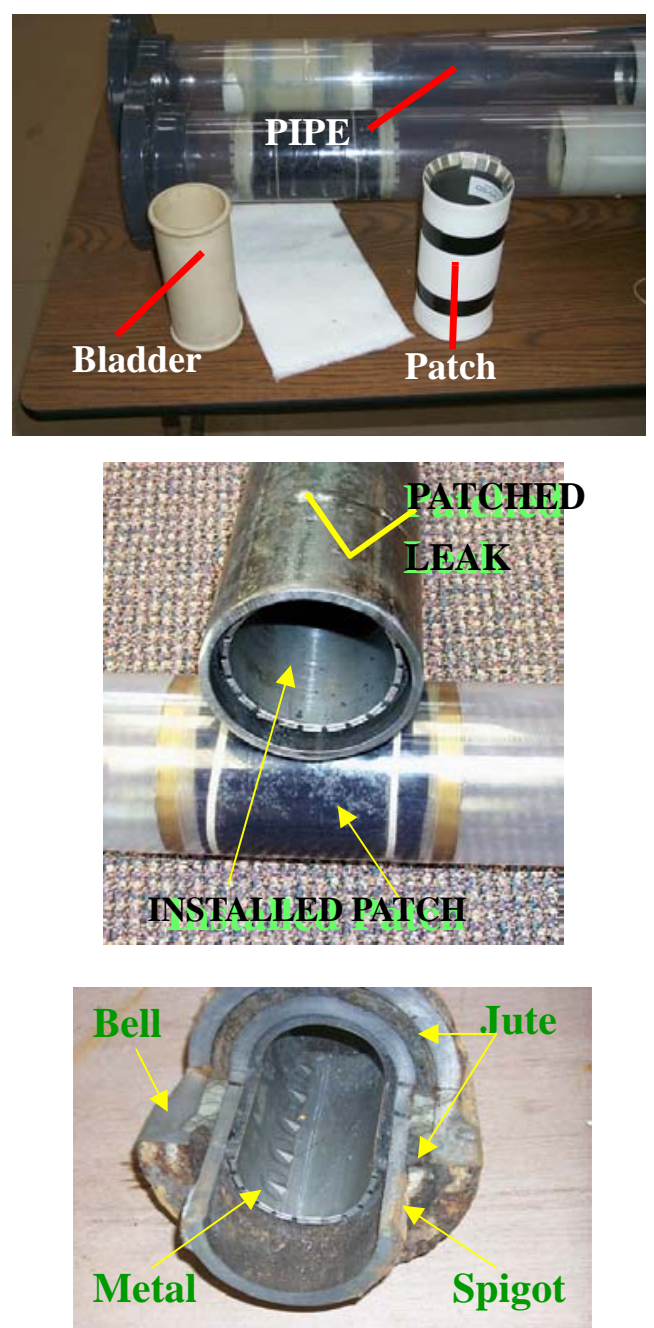

Figure 21 : Sealing patch with modifications and pipe and cast-iron joint installation examples

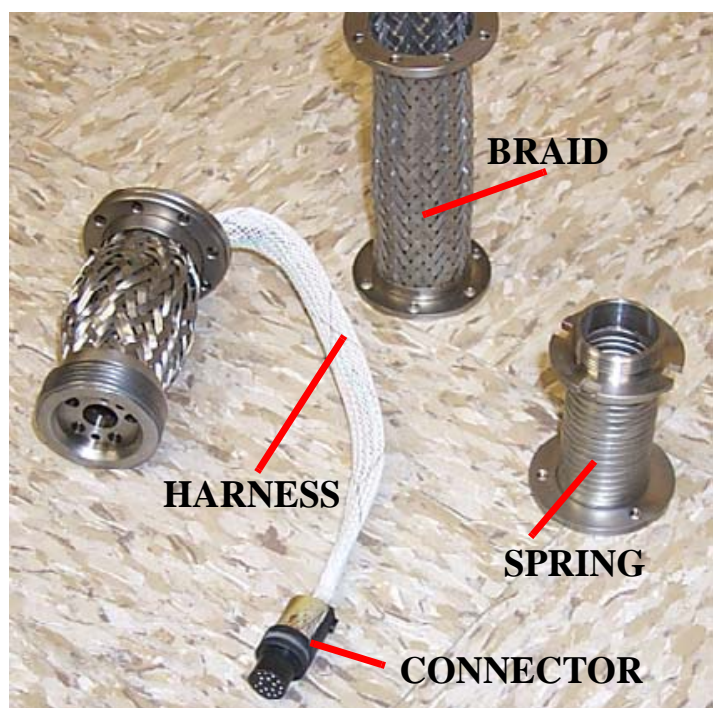

Figure 22 : Intermodule connector prototype assembly
The intermodule connector is used throughout each train to electrically/pneumatically interconnect each module. The connector was designed to withstand push and pullloads up to 3,000 lbs., while retaining flexibility and selfcentering capabilities. This was accomplished by combining an extension-spring and a metallic-mesh sleeve, with end-attached sleeves and fittings. A pressuretolerant and sealed connector-pair internal to each mounting-flange, provides for the necessary interconnectivity. An assembled view of this system is shown in Figure 22.

The user interface developed for the GRISLEE system was split from the hard controls used for the valve-control of the CT-unit, allowing one to control the spool and the levelwind. The module-trains are all controlled from a single rack-mounted panel-box. The video-signal is overlaid with the spool pay-out counter-value and displayed on a monitor as well as recorded via a VCR.

The control box consists primarily of a custom-built CPU that performs all communication tasks with the basemodule via serial communications, and all associated power-regulation from AC to DC for the base-unit. All buttons are read with a digital I/O card, and status messages are displayed on a serial LCD display. Binary outputs control status lights and relays for power, as well as video-switching and safety shut-offs. Both the controlrack and the control-box are shown in Figure 23.
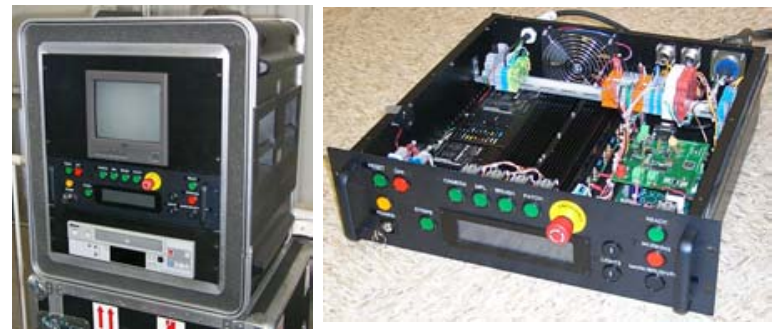

Figure 23 : Control-rack \& control-box for moduletrains

The overall system architecture for the control of GRISLEE is simple (refer to Figure $24 \&$ Figure 25).

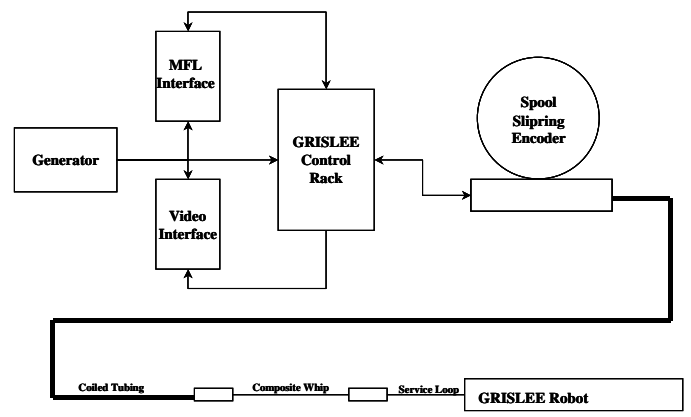

Figure 24 : GRISLEE block-diagram layout 
The system is based on a tethered communications and power backbone that goes through the CT piping and the sliprings, and splits signals for control, MFL-data, video, overlay and control/data into separate conductor-pairs (shielded \& twisted).

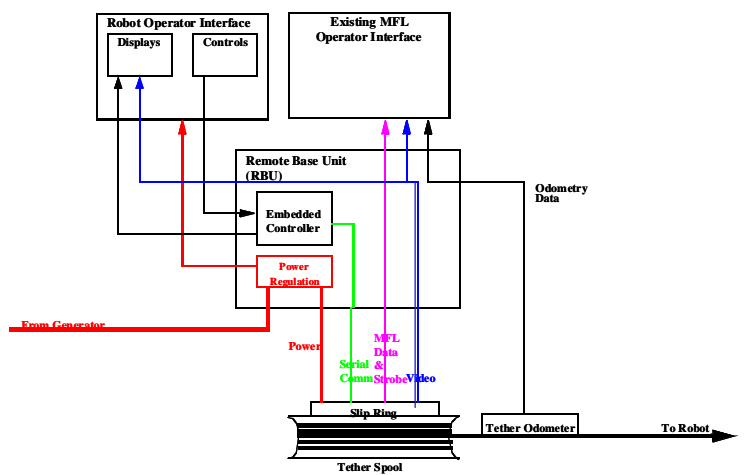

Figure 25 : Data, signal and power diagram

Signals are either processed topside (counter video overlay, operator inputs, base feedback) or in the basemodule (topside commands), or as in the case of the MFL, passed straight through to the dedicated MFL processing unit's CPU (not shown in the figures).

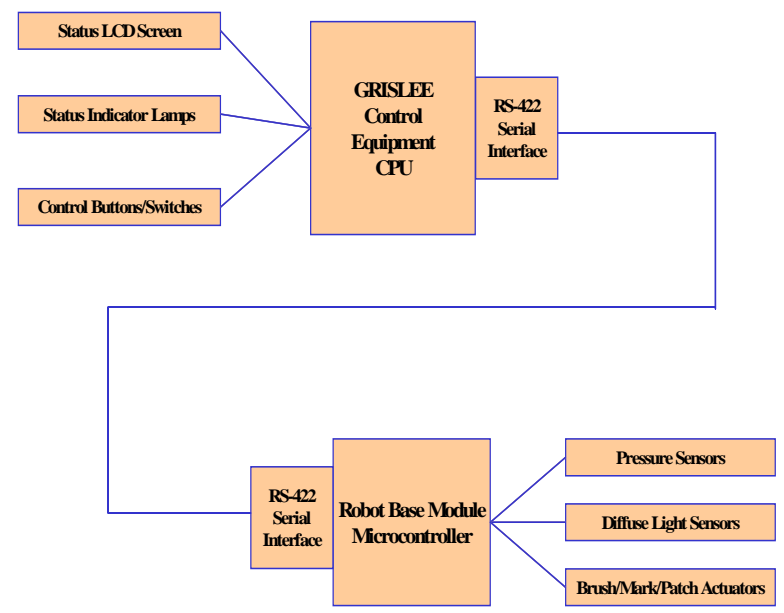

Figure 26: Software event input architecture

The software architecture is based on an interrupt-driven events-table scheme. The topside (control-box CPU) and bottomside (base-module CPU) controllers monitor local events and trigger actions based on these processed readings. A simplified graphic of the architecture is seen in Figure 26. The base-module CPU monitors module-ID values, pressure-sensor thresholds, diffuse-light trigger values, and in return executes digital and analog command sequences for the surface-cleaning motors, lightcontrollers and valve on/off signals. The topside controlbox CPU monitors the feedback coming from the bottomside CPU, as well as buttons and switches, and updates outputs to the LCD screen, the video-overlay board-set and all other button and status indicator lights.

\section{FIELD EVALUATIONS}

The GRISLEE prototype modules/trains and the CT access and deployment system, were demonstrated to the sponsors and utility-industry review committee in an above ground 100-foot long pipe-network set up inside a CMU testing highbay (see Figure 27). The network consisted of several 20-foot steel pipe sections coupled into a 100-foot test-loop, which was pressurized and flowsimulated using compressed air. The demonstration showcased the viability of the live-access and deployment system, the use of the camera- and MFL sensor heads, the marking and locating modules, as well as the surfacepreparation and patch-setting modules. Two patches were set in pre-drilled leaking pipe-sections and joints. These flaws were located, marked, prepared and patched by a minimally-trained operator.

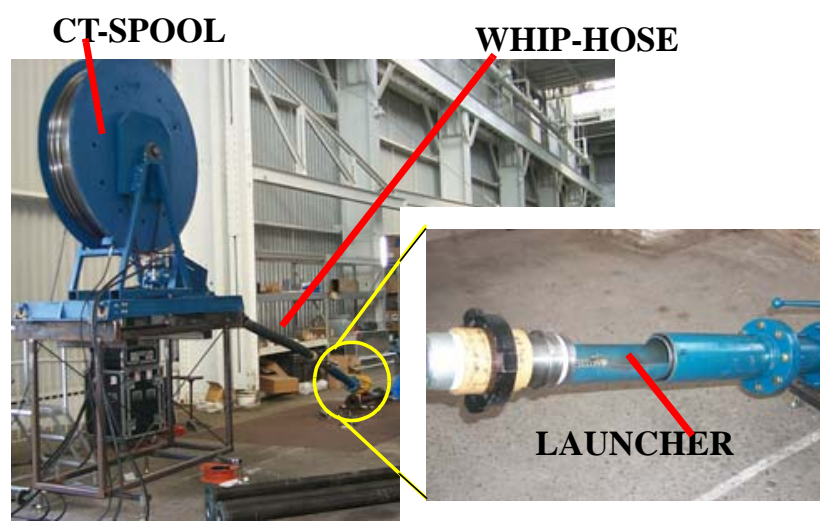

Figure 27 : Prototype-testing setup \& deployment

The step-by-step repair-sequence, the associated infrastructure and logistics, throughput and timing, indicated that the claims of multiple repairs in a live gasmain without flow interruption from a single excavation could be exploited under realistic field conditions. Based on these tests, a list of improvements was drawn up and is being implemented prior to the planned outdoor utility field-trials in late 2,002.

\section{SUMMARY \& CONCLUSIONS}

The GRISLEE and CT systems that were developed for live-access of gas mains and in-situ flaw-detection and spot-repair, were proven in the laboratory-environment. The method of live-access and CT deployment were shown to be reliable, simple and of high throughput with adequate safety margin. The modular GRISLEE design and its ability to assemble dedicated module-trains was proven to be effective in terms of allowing optimal design and achieving desirable performance, enabling piece-part improvements and future expansion. The use of MFL and paint-marking and subsequent positioning via sensordriven stripe-detection was shown to be more than 
adequate. The system proved that mechanical cleaning worked best for joints and pipes. The highly improved patching system, derived from an existing OEM-system, was proven to be feasible in small piping under live conditions with potentially active leak locations.

\section{FUTURE WORK}

The GRISLEE and CT systems are currently being readied for a sequence of utility-sponsored field-trials at several diverse locations within the US. Detailed design documentation and operating and maintenance manuals are also being readied for field-training of utility-crews. The system is expected to complete these trials by late 2,002 .

\section{ACKNOWLEDGEMENTS}

The GRISLEE system was jointly funded at Carnegie Mellon University (CMU) by NASA (under researchgrant \#NCC5-223), and a contract from the Gas Research Institute (\# 5097-290-6029). We wish to further acknowledge the prior development of the MFL sensing technology (by TuboScope Vetco Pipeline Services, Inc.) for distribution gasmains through a jointly-sponsored GRI/ GDF project, as well as separate funding of MEI by GRI, for the development of a modified and upgraded CT deployment system for accessing and deploying GRISLEE into a live gas main.

The GRISLEE system and process have been submitted for national and international patent-filings and have a patent-pending status.

\section{REFERENCES}

[1] Ives, G., Jr., "Pipe Ruptures”, PipeLine \& Gas Industry Journal, Vol. 83, No.9, Houston, TX
[2] Staff Report, "New Optical methane detector improves gas leak surveys", PipeLine \& Gas Industry Journal, September 2000

[3] Porter, C., Pittard, G., "Magnetic Flux Leakage Technology for Inspecting 'Live' Gas-Distribution Mains”, GTI Technical Report \# GRI-99/0199, Oct. 1999, Chicago, IL

[4] Schempf, et al., "Robotic Repair System for Live Distribution Gasmains", Field and Service Robotics Conference., FSR 2001, June 11 - 13, Helsinki, Finland.

[5] Guidance Manual for Operators of Small Natural Gas Systems, US Department of Transportation, Research and Special Programs Administration, August 1997.

[6] Kolesnik, Marina; Baratoff, Gregory, "A Hybrid Model-Based Vision System for Autonomous Navigation of a Sewer Inspection Robot, ERCIM News; (2000), Nr. 42, S. 25-26

[7] Product Literature, Pearpoint, Flexiprobe ELS, www.pearpoint.com

[8] Product Literature, Everest VIT, Rovver, www.everestvit.com

[9] Product Literature, KA-TE, Lateral Main Repair Robot, www.safrdig.com

[10] Product Literature, ARIES, GasCam System, www.ariesind.com/gascam.html

[11] Product Literature, Baker Hughes, VertiLine System,www.bakerhughes.com/bakerhughes/ pipeline_management/pmg_products.htm

[12] Product Literature, Engineering Services, Inc., RISP Robot, www.esit.com/automation/risp.html

[13] Product Literature, LinkPipe, Inc., Ratchet Gas Sealer Sleeve, www.linkpipe.com

[14] Pipeline Repair Robot System, US. Pat \#5,878,783, British Gas/Advantica, www.advanticatech.com

[15] Andrews, M.E., "Large Diameter Sewer Condition Assessment Using Combined Sonar And CCTV Equipment", APWA International Public Works Congress, NRCC/CPWA Seminar Series Innovations in Urban Infrastructure, 2001, Montreal, Canada, www.nrc.ca/irc 